\title{
A New Result of Stability for Thermoelastic-Bresse System of Second Sound Related with Forcing, Delay, and Past History Terms
}

\author{
Djamel Ouchenane, ${ }^{1}$ Zineb Khalili, ${ }^{1}$ Fares Yazid, ${ }^{1}$ Mohamed Abdalla $\mathbb{D}^{2,3}$ \\ Bahri Belkacem Cherif ${ }^{\circ},{ }^{4,5}$ and Ibrahim Mekawy ${ }^{5}$ \\ ${ }^{1}$ Laboratory of Pure and Applied Mathematics, University of Laghouat, Algeria \\ ${ }^{2}$ Mathematics Department, Faculty of Science, King Khalid University, Abha 61471, Saudi Arabia \\ ${ }^{3}$ Mathematics Department, Faculty of Science, South Valley University, Qena 83523, Egypt \\ ${ }^{4}$ Preparatory Institute for Engineering Studies in Sfax, Tunisia \\ ${ }^{5}$ Department of Mathematics, College of Sciences and Arts, ArRas, Qassim University, Saudi Arabia \\ Correspondence should be addressed to Bahri Belkacem Cherif; bahi1968@yahoo.com
}

Received 24 March 2021; Revised 8 April 2021; Accepted 12 April 2021; Published 24 April 2021

Academic Editor: Liliana Guran

Copyright (c) 2021 Djamel Ouchenane et al. This is an open access article distributed under the Creative Commons Attribution License, which permits unrestricted use, distribution, and reproduction in any medium, provided the original work is properly cited.

We consider a one-dimensional linear thermoelastic Bresse system with delay term, forcing, and infinity history acting on the shear angle displacement. Under an appropriate assumption between the weight of the delay and the weight of the damping, we prove the well-posedness of the problem using the semigroup method, where an asymptotic stability result of global solution is obtained.

\section{Introduction}

In this work, we considered with the following problem:

$$
\left\{\begin{array}{l}
\rho_{1} \varphi_{t t}-k\left(\varphi_{x}+l w+\psi\right)_{x}-k_{0} l\left(w_{x}-l \varphi\right)+\mu_{1} \varphi_{t}(x, t)+\mu_{2} \varphi_{t}(x, t-\tau)=0 \\
\rho_{2} \psi_{t t}-b \psi_{x x}+k\left(\varphi_{x}+l w+\psi\right)+\int_{0}^{\infty} g(s) \psi_{x x}(x, t-s) d s+\gamma \theta_{x}+f(\psi)=0 \\
\rho_{1} w_{t t}-k_{0}\left(w_{x}-l \varphi\right)_{x}+k l\left(\varphi_{x}+l w+\psi\right)=0 \\
\rho_{3} \theta_{t}+\kappa q_{x}+\gamma \psi_{t x}=0 \\
\alpha q_{t}+\beta q+\kappa \theta_{x}=0
\end{array}\right.
$$

$(x, t) \in(0,1) \times(0, \infty)$, with initial-boundary conditions

$$
\begin{aligned}
\varphi(0, t) & =\varphi_{x}(1, t)=\psi_{x}(0, t)=\psi(1, t)=w_{x}(0, t) \\
& =w(1, t)=\theta(0, t)=q(1, t)=0, t \geq 0,
\end{aligned}
$$

$$
\left\{\begin{array}{l}
\varphi(x, 0)=\varphi_{0}(x), \varphi_{t}(x, 0)=\varphi_{1}(x), x \in(0,1), \\
\psi(x, 0)=\psi_{0}(x), \psi_{t}(x, 0)=\psi_{1}(x), x \in(0,1), \\
w(x, 0)=w_{0}(x), w_{t}(x, 0)=w_{1}(x), x \in(0,1), \\
\theta(x, 0)=\theta_{0}(x), q(x, 0)=q_{0}(x), \\
\varphi_{t}(x, t-\tau)=f_{0}(x, t-\tau),
\end{array}\right.
$$

with $\tau>0$ is a time delay and $\mu_{1}$ and $\mu_{2}$ are positive real numbers. The function $\theta$ is the temperature difference, $q$ is the heat flux, and $\rho_{1}, \rho_{2}, \rho_{3}, k, l, k_{0}, b, \gamma, \kappa, \alpha, \beta$ are positive constants. We use the energy method and assume that the relaxation function $g$ satisfies the following hypotheses:

(G1) $g: \mathbb{R}_{+} \longrightarrow \mathbb{R}_{+}$is a $C^{1}$ function such that

$$
g(0)>0, b-\int_{0}^{\infty} g(s) d s=b-g_{0}=L>0 .
$$

(G2) Let $\zeta$ be a positive constant with

$$
g^{\prime}(t) \leq-\zeta g(t), \forall t \geq 0
$$


and we suppose that the forcing term $f(\psi(x, t))$ satisfies some hypotheses.

(A1) $f: \mathbb{R} \longrightarrow \mathbb{R}$ such that

$$
\left|f\left(\psi^{2}\right)-f\left(\psi^{1}\right)\right| \leq k_{0}\left(\left|\psi^{1}\right|^{\theta}-\left|\psi^{2}\right|^{\theta}\right)\left|\psi^{1}-\psi^{2}\right|
$$

for all $\psi^{1}, \psi^{2} \in \mathbb{R}$,

where $k_{0}>0, \theta>0$.

(A2)

$$
0 \leq \widehat{f}(\psi) \leq f(\psi) \psi \text { for all } \psi \in \mathbb{R}
$$

with

$$
\widehat{f}(z)=\int_{0}^{z} f(s) d s .
$$
sider

Depending on some of the following parameters, we con-

$$
\begin{gathered}
\tilde{\eta}=\left(1-\frac{\alpha k \rho_{3}}{\rho_{1}}\right)\left(\frac{\rho_{1}}{k}-\frac{\rho_{2}}{b}\right)-\frac{\gamma^{2} \alpha}{b}, \\
k=k_{0} .
\end{gathered}
$$

It is well known that, in the single wave equation, if $\mu_{2}=0$ , that is, in the absence of a delay, the energy of system exponentially decays (see, e.g., [1-22]). On the contrary, if $\mu_{1}=0$, that is, there exists only the delay part in the interior, the system becomes unstable.

Bresse system is a mathematical model that describes the vibration of a planar, linear shearable curved beam. The model was first derived by Bresse [23], and it consists of three coupled wave equations given by

$$
\left\{\begin{array}{l}
\rho_{1} \varphi_{t t}=Q_{x}+l N+F_{1}, \\
\rho_{2} \psi_{t t}=M_{x}-Q+F_{2}, \\
\rho_{1} w_{t t}=N_{x}-I Q+F_{3},
\end{array}\right.
$$

where

$$
\begin{aligned}
& N=k_{0}\left(w_{x}-l \varphi\right), \\
& Q=k\left(\varphi_{x}+l w+\psi\right), \\
& M=b \psi_{x} .
\end{aligned}
$$

We use $N, Q$, and $M$ to denote the axial force, the shear force, and the bending moment. By $w, \varphi$, and $\psi$, we are denoting the longitudinal, vertical, and shear angle displacements. Here, $\rho_{1}=\rho A, \rho_{2}=\rho I, b=E I, k_{0}=E A, k=k^{\prime} G A$, and $l=R^{-1}$ (see, e.g., $[23])$.

The Bresse system (10) is more general than the wellknown Timoshenko system where the longitudinal displacement $\omega$ is not considered $l=0$. The reader may refer to, for example, [24-34].

System (10) is an undamped system, and its associated energy remains constant when the time $t$ evolves. To stabilize system (10), many damping terms have been considered by several authors (see, e.g., [1, 35-40]).

In the succeeding text, we will present some works, which studied the stability of the dissipatif Bresse system. The paper [41] was concerned with asymptotic stability of a Bresse system with two frictional dissipations.

$$
\left\{\begin{array}{l}
\rho_{1} \varphi_{t t}-k\left(\varphi_{x}+l w+\psi\right)_{x}-k_{0} l\left(w_{x}-l \varphi\right)=-\gamma_{1} \varphi_{t}, \\
\rho_{2} \psi_{t t}-b \psi_{x x}+k\left(\varphi_{x}+l w+\psi\right)=-\gamma_{2} \psi_{t}, \\
\rho_{1} w_{t t}-k_{0}\left(w_{x}-l \varphi\right)_{x}+l k\left(\varphi_{x}+l w+\psi\right)=0 .
\end{array}\right.
$$

Under the condition of equal speeds of wave propagation, the authors proved that the system is exponentially stable. Otherwise, they show that Bresse system is not exponentially stable. Then, they proved that the solution decays polynomially to zero with optimal decay rate, depending on the regularity of initial data.

There are several works dedicated to the mathematical analysis of the Bresse system. They are mainly concerned with decay rates of solutions of the linear system. This is done by adding suitable damping effects that can be of thermal, viscous, or viscoelastic nature (see for instance [42-44]), among others.

Concerning thermoelastic Bresse system, [37] considered

$$
\left\{\begin{array}{l}
\rho_{1} \varphi_{t t}-k\left(\varphi_{x}+l w+\psi\right)_{x}-k_{0} l\left(w_{x}-l \varphi\right)+l \gamma \theta_{1}=0 \\
\rho_{2} \psi_{t t}-b \psi_{x x}+k\left(\varphi_{x}+l w+\psi\right)+\gamma \theta_{x}=0 \\
\rho_{1} w_{t t}-k_{0}\left(w_{x}-l \varphi\right)_{x}+k l\left(\varphi_{x}+l w+\psi\right)+\gamma \theta_{1 x}=0 \\
\rho_{3} \theta_{t}-\theta_{x x}+\gamma \psi_{t x}=0 \\
\rho_{3} \theta_{1 x}-\theta_{1 x x}+\gamma\left(w_{t x}-l \varphi_{t}\right)=0
\end{array}\right.
$$

together with initial and specific boundary conditions and proved an exponential and only polynomial-type decay stabilities results.

\section{Preliminaries and Well-Posedness}

Firstly, we assume the following hypothesis:

$$
\left|\mu_{2}\right|<\mu_{1} \text {. }
$$

Using semigroup theory, we will prove that systems (1)-(3) are well posed by introducing the following new variable [17].

$$
z(x, \rho, t)=\varphi_{t}(x, t-\tau \rho), x \in(0,1), \rho \in(0,1), t>0 .
$$

Then, we have

$$
\tau z_{t}(x, \rho, t)+z_{\rho}(x, \rho, t)=0 \text { in }(0,1) \times(0,1) \times(0, \infty) .
$$

Further, let

$$
\eta^{t}(x, s)=\psi(x, t)-\psi(x, t-s), s \geq 0
$$


For this reason, we observe that

$$
\eta_{t}^{t}(x, s)+\eta_{s}^{t}(x, s)=\psi_{t}(x, t)
$$

Therefore, problem (1) takes the form

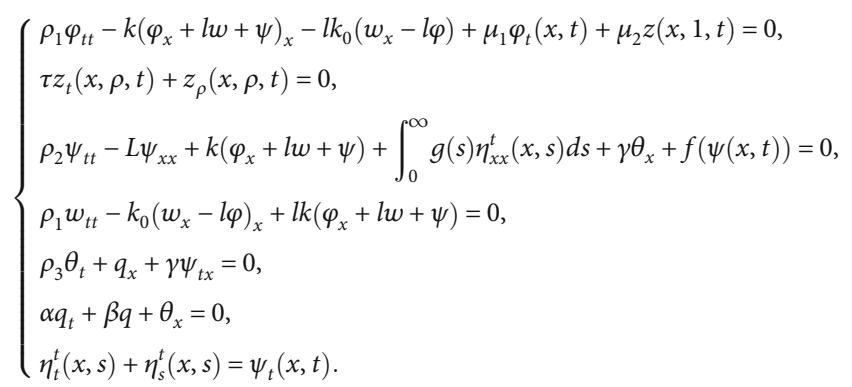

The following are with the boundary conditions:

$$
\begin{aligned}
\varphi(0, t) & =\varphi_{x}(1, t)=\psi_{x}(0, t)=\psi(1, t)=w_{x}(0, t) \\
& =w(1, t)=\theta(0, t)=q(1, t)=0, t \geq 0 .
\end{aligned}
$$

The initial conditions are as follows:

$$
\left\{\begin{array}{l}
\varphi(x, 0)=\varphi_{0}(x), \varphi_{t}(x, 0)=\varphi_{1}(x), \\
\psi(x, 0)=\psi_{0}(x), x \in(0,1), \\
\psi_{t}(x, 0)=\psi_{1}(x), w(x, 0)=w_{0}(x), \\
w_{t}(x, 0)=w_{1}(x), x \in(0,1), \\
\theta(x, 0)=\theta_{0}(x), q(x, 0)=q_{0}(x), x \in(0,1), \\
\varphi_{t}(x,-t)=f_{0}(x, t) \text { in }(0,1) \times(0, \tau), \\
z(x, 1, t)=f(x, t-\tau) \text { in }(0,1) \times(0, \tau), \\
\eta^{t}(x, 0)=0, \forall t \geq 0, \\
\eta^{t}(0, s)=\eta^{t}(1, s)=0 \forall s \geq 0, \\
\eta^{0}(x, s)=\eta_{0}(s)=0 \forall s \geq 0 .
\end{array}\right.
$$

Let $\xi$ be positive constants such that

$$
\tau\left|\mu_{2}\right|<\xi<\tau\left(2 \mu_{1}-\left|\mu_{2}\right|\right)
$$

where $\tau$ is a real number with $0<\tau$ and $\mu_{1}, \mu_{2}$ are a positive constants, and the initial data are $\left(\varphi_{0}, \varphi_{1}, \psi_{0}, \psi_{1}, w_{0}, w_{1}, f\right.$, $\left.\theta_{0}, q_{0}, \eta_{0}\right)$.

If we set

$$
U=\left(\varphi, \varphi_{t}, z, \psi, \psi_{t}, w, w_{t}, \theta, q, \eta^{t}\right)^{T}
$$

then

$$
U^{\prime}=\left(\varphi_{t}, \varphi_{t t}, z_{t}, \psi_{t}, \psi_{t t}, w_{t}, w_{t t}, \theta_{t}, q_{t}, \eta_{t}^{t}\right)^{T}
$$

Therefore, problems (19)-(21) can be written as

$$
\left\{\begin{array}{l}
U^{\prime}(t)=A U(t)+F, \\
U(0)=\left(\varphi_{0}, \varphi_{1}, f_{1}(.-, \tau), \psi_{0}, \psi_{1}, w_{0}, w_{1}, \theta_{0}, q_{0}, \eta_{0}\right),
\end{array}\right.
$$

where the operator $A$ is defined by

$$
A\left(\begin{array}{c}
\varphi \\
u \\
z \\
\psi \\
v \\
w \\
\omega \\
\theta \\
q \\
\phi
\end{array}\right)=\left(\begin{array}{c}
u \\
\frac{k}{\rho_{1}}\left(\varphi_{x}+l w+\psi\right)_{x}+\frac{k_{0} l}{\rho_{1}}\left(w_{x}-l \varphi\right)-\frac{\mu_{1}}{\rho_{1}} u-\frac{\mu_{2}}{\rho_{1}} z(., 1) \\
-\left(\frac{1}{\tau}\right) z_{\rho} \\
v \\
\frac{L}{\rho_{2}} \psi_{x x}-\frac{k}{\rho_{2}}\left(\varphi_{x}+l w+\psi\right)+\frac{1}{\rho_{2}} \int_{0}^{\infty} g(s) \phi_{x x}(s) d s-\frac{\gamma}{\rho_{2}} \theta_{x} \\
\omega \\
\frac{k_{0}}{\rho_{1}}\left(w_{x}-l \varphi\right)_{x}-\frac{k l}{\rho_{1}}\left(\varphi_{x}+l w+\psi\right) \\
-\frac{1}{\rho_{3}} q_{x}-\frac{\gamma}{\rho_{3}} v_{x} \\
-\frac{\beta}{\alpha} q-\frac{k}{\alpha} \theta_{x} \\
-\phi_{s}+v
\end{array}\right),
$$

$$
F=\left(\begin{array}{c}
0 \\
0 \\
0 \\
0 \\
-\frac{1}{\rho_{2}} f(\psi) \\
0 \\
0 \\
0 \\
0 \\
0
\end{array}\right)
$$

We consider the following spaces:

$$
\begin{gathered}
H_{*}^{1}(0,1)=\left\{h \in H^{1}(0,1): h(0)=0\right\}, \\
\tilde{H}_{*}^{1}(0,1)=\left\{h \in H^{1}(0,1): h(1)=0\right\}, \\
H_{*}^{2}(0,1)=H^{2}(0,1) \cap H_{*}^{1}(0,1), \\
\tilde{H}_{*}^{2}(0,1)=H^{2}(0,1) \cap \tilde{H}_{*}^{1}(0,1), \\
\mathscr{H}=H_{*}^{1}(0,1) \times L^{2}(0,1) \times L^{2}\left((0,1), H_{0}^{1}(0,1)\right) \\
\times \tilde{H}_{*}^{1}(0,1) \times L^{2}(0,1) \times \tilde{H}_{*}^{1}(0,1) \times L^{2}(0,1) \\
\times \tilde{H}_{*}^{1}(0,1) \times L^{2}(0,1) \times L^{2}(0,1) \times L^{2}(0,1) \\
\times L_{g}^{2}\left(\mathbb{R}^{+}, H_{0}^{1}(0,1)\right),
\end{gathered}
$$


where $L_{g}^{2}\left(\mathbb{R}^{+}, H_{0}^{1}(0,1)\right)$ denotes the Hilbert space of $H_{0}^{1}-$ valued functions on $\mathbb{R}^{+}$, endowed with the inner product

$$
\left(V_{1}, V_{2}\right)_{L_{g}^{2}\left(\mathbb{R}^{+}, H_{0}^{1}(\Omega)\right)}=\int_{0}^{1} \int_{0}^{1} g(s) V_{1 x}(s) V_{2 x}(s) d s d x
$$

We will show under the assumption (22) that $A$ generates a $C_{0}$ semigroup on $\mathscr{H}$.

Now, we consider the vectors

$$
\begin{aligned}
& U=(\varphi, u, z, \psi, v, w, \oplus, \theta, q, \phi)^{T}, \\
& \bar{U}=(\bar{\varphi}, \bar{u}, \bar{z}, \bar{\psi}, \bar{v}, \bar{w}, \bar{\omega}, \bar{\theta}, \bar{q}, \bar{\phi})^{T},
\end{aligned}
$$

and we define the inner product

$$
\begin{aligned}
\langle U, \bar{U}\rangle_{\mathscr{H}}= & k \int_{0}^{1}\left(\varphi_{x}+\psi+l w\right)\left(\bar{\varphi}_{x}+\bar{\psi}+l \bar{w}\right) d x+\rho_{2} \int_{0}^{1} v \bar{v} d x \\
& +\rho_{1} \int_{0}^{1} \omega \bar{\omega} d x+k_{0} \int_{0}^{1}\left(w_{x}-l \varphi\right)\left(\bar{w}_{x}-l \bar{\varphi}\right) d x \\
& +l \int_{0}^{1} \psi_{x} \bar{\psi}_{x} d x+\rho_{1} \int_{0}^{1} u \bar{u} d x+\xi \int_{0}^{1} \int_{0}^{1} z \bar{z} d \rho d x \\
& +\rho_{3} \int_{0}^{1} \theta \bar{\theta} d x+\alpha \int_{0}^{1} q \bar{q} d x \\
& +\int_{0}^{1} \int_{0}^{\infty} g(s) \phi_{x}(s) \bar{\phi}_{x}(s) d x d s,
\end{aligned}
$$

where the domain of $A$ is defined by

$$
D(A)=\left\{\begin{array}{c}
U \in \mathscr{H} l \varphi \in H_{*}^{2}(0,1) ; \psi, w \in \tilde{H}_{*}^{2}(0,1), u, \theta \in H_{*}^{1}(0,1) ; \\
v, \emptyset, q \in \tilde{H}_{*}^{1}(0,1), u=z(., 0), z_{\rho} \in L^{2}\left((0,1) ; L^{2}(0,1)\right) \\
, \varphi_{x}(1)=0, w_{x}(0)=\psi_{x}(0)=0 \\
\phi_{s} \in L_{g}^{2}\left(\mathbb{R}^{+}, H_{0}^{1}(0,1)\right), \phi(x, 0)=0
\end{array}\right\}
$$

Important properties of the above metrics are stated in the following lemmas. Although most of these results are followed straightforwardly from the known results, they are crucial for what follows. So for the convenience of the reader, we give their proofs here.

Lemma 1. The operator $A$ is dissipative and satisfies, for any $U \in D(A)$,

$$
\begin{aligned}
\langle A U, U\rangle_{\mathscr{H}}= & -\beta \int_{0}^{1} q^{2} d x+\left(-\mu_{1}+\frac{\mu_{2}}{2}+\frac{\xi}{2 \tau}\right) \int_{0}^{1} u^{2} d x \\
& +\left(\frac{\mu_{2}}{2}-\frac{\xi}{2 \tau}\right) \int_{0}^{1} z^{2}(x, 1) d x \\
& +\frac{1}{2} \int_{0}^{1} \int_{0}^{\infty} g^{\prime}(s)\left|\phi_{x}(x, s)\right|^{2} d s d x \leq 0 .
\end{aligned}
$$

Proof. For any $U \in D(A)$, using the inner product,

$$
\langle A U, U\rangle_{\mathscr{H}}=\left\langle\left(\begin{array}{c}
\frac{k}{\rho_{1}}\left(\varphi_{x}+l w+\psi\right)_{x}+\frac{k_{0} l}{\rho_{1}}\left(w_{x}-l \varphi\right)-\frac{\mu_{1}}{\rho_{1}} u-\frac{\mu_{2}}{\rho_{1}} z(., 1) \\
-\left(\frac{1}{\tau}\right) z_{\rho} \\
v \\
\frac{L}{\rho_{2}} \psi_{x x}-\frac{k}{\rho_{2}}\left(\varphi_{x}+l w+\psi\right)+\frac{1}{\rho_{2}} \int_{0}^{\infty} g(s) \phi_{x x}(s) d s-\frac{\gamma}{\rho_{2}} \theta_{x} \\
-\omega \\
\frac{k_{0}}{\rho_{1}}\left(w_{x}-l \varphi\right)_{x}-\frac{k l}{\rho_{1}}\left(\varphi_{x}+l w+\psi\right) \\
-\frac{1}{\rho_{3}} q_{x}-\frac{\gamma}{\rho_{3}} v_{x} \\
-\frac{\beta}{\alpha} q-\frac{1}{\alpha} \theta_{x} \\
-\phi_{s}+v
\end{array}\right),\left(\begin{array}{l}
\varphi \\
u \\
z \\
\psi \\
w \\
\omega \\
\theta \\
q \\
\phi
\end{array}\right)\right\rangle
$$


Then,

$$
\begin{aligned}
\langle A U, U\rangle_{\mathscr{H}}= & k \int_{0}^{1}\left(u_{x}+v+l \Phi\right)\left(\varphi_{x}+l w+\psi\right) d x+k_{0} \int_{0}^{1}\left(\Phi_{x}-l u\right) \\
& \cdot\left(w_{x}-l \varphi\right) d x+k \int_{0}^{1}\left(\varphi_{x}+l w+\psi\right) u d x \\
& +k_{0} l \int_{0}^{1}\left(w_{x}-l \varphi\right) u d x-\mu_{1} \int_{0}^{1} u^{2} d x \\
& -\mu_{2} \int_{0}^{1} z(x, 1) u d x+L \int_{0}^{1} \psi_{x x} v d x \\
& -k \int_{0}^{1}\left(\varphi_{x}+l w+\psi\right) v d x-\gamma \int_{0}^{1} \theta_{x} v d x \\
& +k_{0} \int_{0}^{1}\left(w_{x}-l \varphi\right) \oplus d x-k l \int_{0}^{1}\left(\varphi_{x}+l w+\psi\right) \oplus d x \\
& +L \int_{0}^{1} v_{x} \psi_{x} d x+\int_{0}^{1} \int_{0}^{\infty} g(s) \phi_{x}(s)\left(-\phi_{s}+v\right) d x d s \\
& -\int_{0}^{1} q_{x} \theta d x-\gamma \int_{0}^{1} u_{x} \theta d x-\beta \int_{0}^{1} q^{2} d x-\int_{0}^{1} \theta_{x} q d x \\
& -\xi \int_{0}^{1} \int_{0}^{1} z z_{\rho} d \rho d x .
\end{aligned}
$$

By the fact that

$$
\begin{aligned}
-\beta \int_{0}^{1} q^{2} d x-\mu_{1} \int_{0}^{1} u^{2} d x-\mu_{2} \int_{0}^{1} z(x, 1) u d x+\int_{0}^{1} \int_{0}^{\infty} g(s) \phi_{x}(s) \\
\cdot\left(-\phi_{s}+v\right) d x d s-\frac{\xi}{\tau} \int_{0}^{1} \int_{0}^{1} z(x, \rho) z_{\rho}(x, \rho) d \rho d x \\
=-\beta \int_{0}^{1} q^{2} d x-\mu_{1} \int_{0}^{1} u^{2} d x-\mu_{2} \int_{0}^{1} z(x, 1) u d x+\int_{0}^{1} \int_{0}^{\infty} g(s) \phi_{x}(s) \\
\cdot\left(-\phi_{s}+v\right) d x d s-\frac{\xi}{2 \tau} \int_{0}^{1} \int_{0}^{1} \frac{\partial}{\partial \rho} z^{2}(x, \rho) d \rho d x \\
=-\beta \int_{0}^{1} q^{2} d x-\mu_{1} \int_{0}^{1} u^{2} d x-\mu_{2} \int_{0}^{1} z(x, 1) u d x+\int_{0}^{1} \int_{0}^{\infty} g(s) \phi_{x}(s) \\
\cdot\left(-\phi_{s}+v\right) d x d s-\frac{\xi}{2 \tau} \int_{0}^{1}\left\{z^{2}(x, 1)-z^{2}(x, 0)\right\} d x \\
=-\beta \int_{0}^{1} q^{2} d x-\mu_{1} \int_{0}^{1} u^{2} d x-\mu_{2} \int_{0}^{1} z(x, 1) u d x+\int_{0}^{1} \int_{0}^{\infty} g(s) \phi_{x}(s) \\
\cdot\left(-\phi_{s}+v\right) d x d s-\frac{\xi}{2 \tau} \int_{0}^{1} z^{2}(x, 1) d x+\frac{\xi}{2 \tau} \int_{0}^{1} u^{2} d x,
\end{aligned}
$$

and using Young's inequality, we find

$$
\begin{aligned}
\langle A U, U\rangle_{H} \leq & -\beta \int_{0}^{1} q^{2} d x+\left(-\mu_{1}+\frac{\mu_{2}}{2}+\frac{\xi}{2 \tau}\right) \int_{0}^{1} u^{2} d x \\
& +\left(\frac{\mu_{2}}{2}-\frac{\xi}{2 \tau}\right) \int_{0}^{1} z^{2}(x, 1) d x \\
& +\frac{1}{2} \int_{0}^{1} \int_{0}^{\infty} g^{\prime}(s)\left|\phi_{x}(x, s)\right|^{2} d s d x
\end{aligned}
$$

Keeping in mind condition (22), the desired result yields.
Lemma 2. The operator $I-A$ is surjective.

Proof. We need to show that for all $\mathscr{F}=\left(f_{1}, f_{2}, f_{3}, f_{4}, f_{5}, f_{6}\right.$, $\left.f_{7} f_{8}, f_{9}, f_{10}\right)^{T} \in \mathscr{H}$, there exists $U \in D(A)$ such that

$$
U-A U=\mathscr{F},
$$

that is,

$$
\left\{\begin{array}{l}
-u+\varphi=f_{1} \in H_{*}^{1}(0,1), \\
-k\left(\varphi_{x}+l w+\psi\right)_{x}-k_{0} l\left(w_{x}-l \varphi\right)+\rho_{1} u+\mu_{1} u+\mu_{2} z(., 1)=\rho_{1} f_{2} \in L^{2}(0,1), \\
z+\tau^{-1} z_{\rho}=\tau f_{3} \in L^{2}\left((0,1), H^{1}(0,1)\right), \\
-v+\psi=f_{4} \in \tilde{H}_{*}^{1}(0,1), \\
-L \psi_{x x}+k\left(\varphi_{x}+l w+\psi\right)+\rho_{2} v-\int_{0}^{\infty} g(s) \phi_{x x}(s) d s+\gamma \theta_{x}=\rho_{2} f_{5} \in L^{2}(0,1), \\
-\emptyset+w=f_{6} \in \tilde{H}_{*}^{1}(0,1), \\
-k_{0}\left(w_{x}-l \varphi\right)_{x}+k l\left(\varphi_{x}+l w+\psi\right)+\rho_{1} \varrho=\rho_{1} f_{7} \in L^{2}(0,1), \\
q_{x}+\gamma v_{x}+\rho_{3} \theta=\rho_{3} f_{8} \in L^{2}(0,1), \\
(\beta+\alpha) q+\theta_{x}=\alpha f_{9} \in L^{2}(0,1), \\
\phi+\phi_{s}-v=f_{10} \in L^{2}(0,1) .
\end{array}\right.
$$

From (39), we define

$$
\theta=\frac{\alpha}{k} \int_{0}^{x} f_{9}(y) d y-\frac{\alpha}{k}(\beta+\alpha) \int_{0}^{x} q(y) d y,
$$

so $\theta(0, t)=0$.

Inserting $u=\varphi-f_{1}, v=\psi-f_{4}, \Phi=w-f_{6}$ and (39) into (40), we get

$$
\left\{\begin{array}{l}
-k\left(\varphi_{x}+l w+\psi\right)_{x}-k_{0} l\left(w_{x}-l \varphi\right)+\rho_{1} \varphi+\mu_{1} u+\mu_{2} z(., 1)=h_{1} \in L^{2}(0.1), \\
-L \psi_{x x}+k\left(\varphi_{x}+l w+\psi\right)+\rho_{2} \psi-\int_{0}^{\infty} g(s) \phi_{x x}(s) d s-\gamma(\beta+\alpha) q=h_{2} \in L^{2}(0.1), \\
-k_{0}\left(w_{x}-l \varphi\right)_{x}+k l\left(\varphi_{x}+l w+\psi\right)+\rho_{1} w=h_{3} \in L^{2}(0.1) \\
q_{x}+(\beta+\alpha) \int_{0}^{x} q(y) d y-\gamma \psi_{x}=h_{4} \in L^{2}(0.1) \\
z+\tau^{-1} z_{\rho}=h_{5} \in L^{2}(0.1) \\
\phi+\phi_{s}-v=h_{6} \in L^{2}(0.1)
\end{array}\right.
$$

where

$$
\left\{\begin{array}{l}
h_{1}=\rho_{1}\left(f_{1}+f_{2}\right), \\
h_{2}=\rho_{2}\left(f_{4}+f_{5}\right)-\frac{\alpha}{k} \gamma f_{9}, \\
h_{3}=\rho_{1}\left(f_{6}+f_{7}\right) \\
h_{4}=\gamma f_{4 x}+\rho_{3}\left(f_{8}-\frac{\alpha}{k} \int_{0}^{x} f_{9}(y) d y\right), \\
h_{5}=z+\tau^{-1} z_{\rho}, \\
h_{6}=\phi+\phi_{s}-v .
\end{array}\right.
$$


Furthermore, by (39), we can find as $z(x, 0)=u(x)$ for $x \in(0,1)$. Following the same last approach, we obtain by using equation for $z$ in (39)

$$
z(x, \rho)=u(x) e^{-\tau \rho}+\tau e^{-\tau \rho} \int_{0}^{\rho} f_{3}(x, s) e^{\tau \rho s} d s .
$$

From (39), we obtain

$$
z(x, \rho)=\varphi(x) e^{-\tau \rho}-f_{1} e^{-\tau \rho}+\tau e^{-\tau \rho} \int_{0}^{\rho} f_{3}(x, s) e^{\tau \rho s} d s
$$

Then,

$$
z(x, 1)=\varphi(x) e^{-\tau}+z_{0}(x)
$$

such that

$$
z_{0}(x)=-f_{1} e^{-\tau}+\tau e^{-\tau} \int_{0}^{\rho} f_{3}(x, s) e^{\tau s} d s .
$$

We note that the last equation in (41) with $\phi(x, 0)=0$ has a unique solution

$$
\begin{aligned}
\phi(x, s) & =\left(\int_{0}^{x} e^{y}\left(f_{10}(x, y)+v(x) d y\right) e^{-s}\right) \\
& =\left(\int_{0}^{x} e^{y}\left(f_{10}(x, y)+\psi(x)-f_{4}(x) d y\right) e^{-s}\right) .
\end{aligned}
$$

In order to solve (42), we consider

$$
a((\varphi, \psi, w, q),(\tilde{\varphi}, \tilde{\psi}, \tilde{w}, \tilde{q}))=L(\tilde{\varphi}, \tilde{\psi}, \tilde{w}, \tilde{q})
$$

where

$$
a:\left[H_{*}^{1}(0,1) \times \tilde{H}_{*}^{1}(0,1) \times \tilde{H}_{*}^{1}(0,1) \times L^{2}(0,1)\right]^{2} \longrightarrow \mathbb{R}
$$

is the bilinear form given by

$$
\begin{aligned}
a((\varphi, \psi, w, q),(\tilde{\varphi}, \tilde{\psi}, \tilde{w}, \tilde{q})) & \\
= & k \int_{0}^{1}\left(\varphi_{x}+l w+\psi\right)\left(\tilde{\varphi}_{x}+l \tilde{w}+\tilde{\psi}\right) d x+(\beta+\alpha) \int_{0}^{1} q \tilde{q} d x \\
& +b \int_{0}^{1} \psi_{x} \tilde{\psi}_{x} d x+\rho_{2} \int_{0}^{1} \psi \tilde{\psi} d x-\gamma(\beta+\alpha) \int_{0}^{1} q \tilde{\psi} d x \\
& +\rho_{1} \int_{0}^{1} \psi \tilde{\psi} d x+\gamma(\beta+\alpha) \int_{0}^{1} \psi \tilde{q} d x+\rho_{1} \int_{0}^{1} w \tilde{w} d x \\
& +k_{0} \int_{0}^{1}\left(w_{x}-l \varphi\right)\left(\tilde{w}_{x}-l \tilde{\varphi}\right) d x+\int_{0}^{1} \varphi \tilde{\varphi}\left(\mu_{1}+\mu_{2} e^{-\tau}\right) d x \\
& +\rho_{3}(\beta+\alpha) \int_{0}^{1}\left(\int_{0}^{x} q(y) d y \int_{0}^{x} \tilde{q}(y) d y\right) d x . \\
L: & \left.H_{*}^{1}(0,1) \times \tilde{H}_{*}^{1}(0,1) \times \tilde{H}_{*}^{1}(0,1) \times L^{2}(0,1)\right] \longrightarrow \mathbb{R}
\end{aligned}
$$

is the linear form defined by

$$
\begin{aligned}
L(\tilde{\varphi}, \tilde{\psi}, \tilde{w}, \tilde{q})= & \int_{0}^{1} h_{1} \tilde{\varphi} d x+\int_{0}^{1} h_{2} \tilde{\psi} d x+\int_{0}^{1} h_{3} \tilde{w} d x \\
& +(\alpha+\beta) \int_{0}^{1} h_{4} \int_{0}^{x} \tilde{q}(y) d y d x+\int_{0}^{1}\left(\mu_{1} f_{1} \mu_{2} z_{0}\right) \tilde{\varphi} d x
\end{aligned}
$$

It is easy to verify that $a$ is continuous and coercive, and $L$ is continuous. So applying the Lax-Milgram theorem, we deduce that for all $(\tilde{\varphi}, \tilde{\psi}, \tilde{w}, \tilde{q}) \in H_{*}^{1}(0,1) \times \tilde{H}_{*}^{1}(0$, 1) $\times \tilde{H}_{*}^{1}(0,1) \times L^{2}(0,1)$, problem $(48)$ admits a unique solution $(\varphi, \psi, w, q) \in H_{*}^{1}(0,1) \times \tilde{H}_{*}^{1}(0,1) \times \tilde{H}_{*}^{1}(0,1) \times L^{2}(0,1)$. Since $D(A)$ is dense in $\mathscr{H}$ consequently, using Lemmas 1 and 2, we conclude that $A$ is a maximal monotone operator. Hence, by Hille-Yosida theorem (see [45]), we have the following well-posedness result such that (25) is satisfied.

Theorem 3. Let $U_{0} \in \mathscr{H}$, then there exists a unique weak solution $U \in C\left(\mathbb{R}^{+}, \mathscr{H}\right)$ of problems (1)-(3). Moreover, if $U_{0} \in D$ $(A)$, then $U \in C\left(\mathbb{R}^{+}, D(A)\right) \cap C^{l}\left(\mathbb{R}^{+}, \mathscr{H}\right)$.

Lemma 4. The operator F defined in (26) is locally Lipschitz in $\mathscr{H}$.

Proof. Let $U=(\varphi, u, z, \psi, v, w, \omega, \theta, q, \phi)^{T}, \bar{U}=(\bar{\varphi}, \bar{u}, \bar{z}, \bar{\psi}, \bar{v}$, $\bar{w}, \bar{\omega}, \bar{\theta}, \bar{q}, \bar{\phi})^{T}$, then we have

$$
\|F(U)-F(\bar{U})\|_{\mathscr{H}} \leq\|f(\psi)-f(\bar{\psi})\|_{L^{2}}
$$

By using (6), Holder's and Poincaré's inequalities, we can obtain

$$
\|f(\psi)-f(\bar{\psi})\|_{L^{2}} \leq\left(\|\psi\|_{2 \theta}^{\theta}+\|\bar{\psi}\|_{2 \theta}^{\theta}\right)\|\psi-\bar{\psi}\| \leq c_{1}\|\psi-\bar{\psi}\|,
$$

which gives us

$$
\|F(U)-F(\bar{U})\|_{\mathscr{H}} \leq c_{1}\|\psi-\bar{\psi}\|_{\mathscr{H}} .
$$

Then, the operator $F$ is locally Lipschitz in $\mathscr{H}$. The proof is hence complete.

\section{Exponential Stability}

Here, we present our stability result for the energy of the solution of systems (1)-(3), by using the multiplier technique. So we define the energy of our system by 


$$
\begin{aligned}
E(t)= & \frac{1}{2} \int_{0}^{1}\left[\rho_{1} \varphi_{t}^{2}+\rho_{2} \psi_{t}^{2}+\rho_{1} w_{t}^{2}+b \psi_{x}^{2}+\rho_{3} \theta^{2}+\alpha q^{2}\right. \\
& \left.+k\left(\varphi_{x}+\psi+l w\right)^{2}+k_{0}\left(w_{x}-l \varphi\right)^{2}\right] d x \\
& +\frac{\xi}{2} \int_{0}^{1} \int_{0}^{1} z^{2}(x, \rho, t) d \rho d x+\frac{1}{2} \int_{0}^{1} \int_{0}^{\infty} g(s)\left|\eta_{x}^{t}(x, s)\right|^{2} d s d x \\
& +\int_{0}^{1} \widehat{f}(\psi(t)) d x .
\end{aligned}
$$

The proof of the stability for our system is based on the following lemmas:

Lemma 5. Let $\left(\varphi, \psi, w, \theta, q, z, \eta^{t}\right)$ be the solution of (19)-(21). Then, the energy functional, defined by (55), satisfies

$$
\begin{aligned}
E^{\prime}(t) \leq & -\beta \int_{0}^{1} q^{2} d x-C \int_{0}^{1} \psi_{t}^{2} d x-\left(\mu_{1}-\frac{\xi}{2 \tau}-\frac{\left|\mu_{2}\right|}{2}\right) \\
& \cdot\left\|\varphi_{t}\right\|_{2}^{2}-\left(\frac{\xi}{2 \tau}-\frac{\left|\mu_{2}\right|}{2}\right)\|z(x, 1, t)\|_{2}^{2} \\
& +\frac{1}{2} \int_{0}^{1} \int_{0}^{\infty} g^{\prime}(s)\left|\eta_{x}^{t}(x, s)\right|^{2} d s d x,
\end{aligned}
$$

such that $C>0$.

Proof. Multiplying $(1.1)_{1},(1.1)_{2},(1.1)_{3},(1.1)_{4}$, and $(1.1)_{5}$ by $\varphi_{t}, \psi_{t}, w_{t}, \theta$, and $q$, respectively, and after simplification, we have (56).

With the fact

$$
\frac{d}{d t} \widehat{f}(\psi)=f(\psi) \psi
$$

it gives us (56).

Lemma 6. Let $\left(\varphi, \psi, w, \theta, q, z, \eta^{t}\right)$ be the solution of (19)-(21). We have

$$
F_{1}(t):=\alpha \rho_{3} \int_{0}^{1} \theta \int_{0}^{x} q(y) d y d x
$$

satisfies, for any $\varepsilon_{1}>0$, the estimate

$$
F_{1}^{\prime}(t) \leq-\frac{\rho_{3}}{2} \int_{0}^{1} \theta^{2} d x+\varepsilon_{1} \int_{0}^{1} \psi_{t}^{2} d x+c\left(1+\frac{1}{\varepsilon_{1}}\right) \int_{0}^{1} q^{2} d x .
$$

Proof. Taking the derivative of $F_{1}$, using the fourth and fifth equations in (1) and performing integration by parts, we get

$$
\begin{aligned}
F_{1}^{\prime}(t)= & -\rho_{3} k \int_{0}^{1} \theta^{2} d x-\alpha k \int_{0}^{1} q^{2} d x-\alpha \gamma \int_{0}^{1} \psi_{t x} \int_{0}^{1} q(y) d y d x \\
& -\beta \rho_{3} \int_{0}^{1} \theta \int_{0}^{x} q(y) d y d x .
\end{aligned}
$$

According to Cauchy-Schwarz and Young's inequalities with $\varepsilon_{1}>0$, we get (59).

Lemma 7. Let $\left(\varphi, \psi, w, \theta, q, z, \eta^{t}\right)$ be the solution of (19)-(21). We have

$$
F_{2}(t):=-\frac{\rho_{2} \rho_{3}}{\gamma} \int_{0}^{1} \theta \int_{0}^{x} \psi_{t}(y) d y d x
$$

satisfies, for any $\varepsilon_{1}, \varepsilon_{2}, \delta_{1}>0$, the estimate

$$
\begin{aligned}
F_{2}^{\prime}(t) \leq & -\frac{\rho_{2}}{\gamma} \int_{0}^{1} \psi_{t}^{2} d x+\varepsilon_{2} \int_{0}^{1}\left(\varphi_{x}+\psi+l w\right)^{2} d x \\
& +\left(\varepsilon_{3}+\frac{\rho_{3}}{\gamma}\left(\frac{\varepsilon_{2}}{b^{2} \lambda_{2}}+\frac{b^{2}}{2 \varepsilon_{2} \lambda_{2}}\right)\right) \int_{0}^{1} \psi_{x}^{2} d x \\
& +c\left(1+\frac{1}{\varepsilon_{2}}+\frac{1}{\varepsilon_{3}}\right) \int_{0}^{1} \theta^{2} d x+c \int_{0}^{1} q^{2} d x \\
& +\frac{g_{0}}{4 \delta_{1}} \int_{0}^{1} \int_{0}^{\infty} g(s)\left|\eta_{x}^{t}(x, s)\right|^{2} d s d x . \\
& +\left(\delta_{1}+C_{1}\right) \int_{0}^{1} \theta_{x}^{2} d x .
\end{aligned}
$$

Proof. For differentiation of $F_{2}$, using equations in (1) and integration by parts, we obtain

$$
\begin{aligned}
F_{2}^{\prime}(t)= & -\rho_{2} \int_{0}^{1} \psi_{t}^{2} d x-\frac{\rho_{2} k}{\gamma} \int_{0}^{1} q \psi_{t} d x+\rho_{3} \int_{0}^{1} \theta^{2} d x \\
& -\frac{b \rho_{3}}{\gamma} \int_{0}^{1} \theta \psi_{x} d x+\frac{k \rho_{3}}{\gamma} \int_{0}^{1}\left(\varphi_{x}+\psi+l w\right) \int_{0}^{x} \theta(y) d y d x \\
& +\frac{\rho_{3}}{\gamma} \int_{0}^{1} \int_{0}^{\infty} g(s) \eta_{x}^{t}(x, s) d s \int_{0}^{x} \theta_{x}(y) d y d x \\
& +\frac{\rho_{3}}{\gamma} \int_{0}^{1} \theta \int_{0}^{x} f(\psi) d y d x .
\end{aligned}
$$

Estimate (62) follows by using Cauchy-Schwarz, Young's, and Poincarés inequalities that

$$
\begin{aligned}
\int_{0}^{1}|f(\psi) \theta| d x & \leq \int_{0}^{1}|\psi|^{\theta}|\psi||\theta| d x \leq\|\psi\|_{2(\theta+1)}^{\theta}\|\psi\|_{2(\theta+1)}\|\theta\| \\
& \leq C_{1} \int_{0}^{1} \theta^{2} d x
\end{aligned}
$$

Lemma 8. Let $\left(\varphi, \psi, w, \theta, q, z, \eta^{t}\right)$ be the solution of (19)-(21). Then, the energy functional

$$
F_{3}(t):=-\rho_{1} \int_{0}^{1}\left(\varphi \varphi_{t}+w w_{t}\right) d x
$$


satisfies the estimate

$$
\begin{aligned}
F_{3}^{\prime}(t) \leq & -\left(\rho_{1}-\frac{1}{4 \varepsilon_{4}}\right) \int_{0}^{1} \varphi_{t}^{2} d x+c \int_{0}^{1} \psi_{x}^{2} d x+k_{0} \int_{0}^{1}\left(w_{x}-l \varphi\right)^{2} d x \\
& +c \int_{0}^{1}\left(\varphi_{x}+\psi+l w\right)^{2} d x-\rho_{1} \int_{0}^{1} w_{t}^{2} d x \\
& +\left(\varepsilon_{5} \mu_{2}+\mu_{1} \varepsilon_{4}\right) \int_{0}^{1} \varphi^{2} d x+\frac{\mu_{2}}{4 \varepsilon_{5}} \int_{0}^{1} z^{2}(x, 1, t) d x .
\end{aligned}
$$

Proof. Using (1)-(3) gives

$$
\begin{aligned}
F_{3}^{\prime}(t)= & -\rho_{1} \int_{0}^{1} \varphi_{t}^{2} d x+k \int_{0}^{1}\left(\varphi_{x}+\psi+l w\right)^{2} d x \\
& -k \int_{0}^{1}\left(\varphi_{x}+\psi+l w\right) \psi d x-\rho_{1} \int_{0}^{1} w_{t}^{2} d x \\
& +k_{0} \int_{0}^{1}\left(w_{x}-l \varphi\right)^{2} d x+\mu_{1} \int_{0}^{1} \varphi \varphi_{t} d x \\
& +\mu_{2} \int_{0}^{1} \varphi z(x, 1, t) d x .
\end{aligned}
$$

Using Young's and Poincaré's inequalities, estimate (66) is established.

Lemma 9. Let $\left(\varphi, \psi, w, \theta, q, z, \eta^{t}\right)$ be the solution of (19)-(21). Then, the energy functional

$$
F_{4}(t):=\rho_{2} \int_{0}^{1} \psi \psi_{t} d x
$$

satisfies for any $\delta_{2}>0$ the estimate

$$
\begin{aligned}
F_{4}^{\prime}(t) \leq & \left(\frac{b}{2}+\delta_{2}+C_{2}\right) \int_{0}^{1} \psi_{x}^{2} d x+\rho_{2} \int_{0}^{1} \psi_{t}^{2} d x \\
& +\frac{k^{2}}{b} \int_{0}^{1}\left(\varphi_{x}+\psi+l w\right)^{2} d x+c \int_{0}^{1} \theta^{2} d x \\
& +\frac{g_{0}}{4 \delta_{2}} \int_{0}^{1} \int_{0}^{\infty} g(s)\left|\eta_{x}^{t}(x, s)\right|^{2} d s d x .
\end{aligned}
$$

Proof. Taking the derivative of $F_{4}$ and using the second equation in (1), it follows that

$$
\begin{aligned}
F_{4}^{\prime}(t)= & -b \int_{0}^{1} \psi_{x}^{2} d x+\rho_{2} \int_{0}^{1} \psi_{t}^{2} d x+\gamma \int_{0}^{1} \psi_{x} \theta d x \\
& -k \int_{0}^{1}\left(\varphi_{x}+\psi+l w\right) d x \\
& +\int_{0}^{1} \psi_{x}(x) \int_{0}^{\infty} g(s) \eta_{x}^{t}(x, s) d s d x-\int_{0}^{1} \psi f(\psi) d x,
\end{aligned}
$$

$$
\begin{aligned}
\int_{0}^{1}|f(\psi) \psi| d x & \leq \int_{0}^{1}|\psi|^{\theta}|\psi||\psi| d x \leq\|\psi\|_{2(\theta+1)}^{\theta}\|\psi\|_{2(\theta+1)}\|\psi\| \\
& \leq C_{2} \int_{0}^{1} \psi_{x}^{2} d x .
\end{aligned}
$$

Young's and Poincaré's inequalities for (70) yield (69).

Lemma 10. Let $\left(\varphi, \psi, w, \theta, q, z, \eta^{t}\right)$ be the solution of (19)-(21). Then, the energy functional

$$
F_{5}(t):=-\rho_{1} \int_{0}^{1} \varphi_{t}\left(w_{x}-l \varphi\right) d x-\rho_{1} \int_{0}^{1} w_{t}\left(\varphi_{x}+\psi+l w\right) d x
$$

satisfies the estimate

$$
\begin{aligned}
F_{5}^{\prime}(t) \leq & -\left(l k_{0}-\frac{\mu_{1}}{4 \varepsilon_{6}}-\frac{\mu_{2}}{4 \varepsilon_{7}}\right) \int_{0}^{1}\left(w_{x}-l \varphi\right)^{2} d x-\frac{l \rho_{1}}{2} \int_{0}^{1} w_{t}^{2} d x \\
& +\left(l \rho_{1}+\varepsilon_{6} \mu_{1}\right) \int_{0}^{1} \varphi_{t}^{2} d x+c \int_{0}^{1} \psi_{t}^{2} d x \\
& +l k \int_{0}^{1}\left(\varphi_{x}+\psi+l w\right)^{2} d x+\varepsilon_{7} \mu_{2} \int_{0}^{1} z^{2}(x, 1, t) d x .
\end{aligned}
$$

Proof. For differentiation of $F_{5}$, using $(1.1)_{1}$ and $(1.1)_{3}$, we arrive at

$$
\begin{aligned}
F_{5}^{\prime}(t)= & -l k_{0} \int_{0}^{1}\left(w_{x}-l \varphi\right)^{2} d x-l \rho_{1} \int_{0}^{1} w_{t}^{2} d x+l \rho_{1} \int_{0}^{1} \varphi_{t}^{2} d x \\
& +l k \int_{0}^{1}\left(\varphi_{x}+\psi+l w\right)^{2} d x-\rho_{1} \int_{0}^{1} \psi_{t} w_{t} d x \\
& +\mu_{1} \int_{0}^{1} \varphi_{t}\left(w_{x}-l \varphi\right) d x+\mu_{2} \int_{0}^{1} z(x, 1, t)\left(w_{x}-l \varphi\right) d x
\end{aligned}
$$

Young's inequality for (74) yields (73).

Lemma 11. Let $\left(\varphi, \psi, w, \theta, q, z, \eta^{t}\right)$ be the solution of (19)-(21) and let $k=k_{0}$. Then, the functional

$$
\begin{aligned}
F_{6}(t):= & -\rho_{1} \int_{0}^{1}\left(w_{x}-l \varphi\right) \int_{0}^{x} w_{t}(y) d y d x \\
& -\rho_{1} \int_{0}^{1} \varphi_{t} \int_{0}^{x}\left(\varphi_{x}+\psi+l w\right) d y d x
\end{aligned}
$$

satisfies the estimate

$$
\begin{aligned}
F_{6}^{\prime}(t) \leq & -\frac{\rho_{1}}{2} \int_{0}^{1} \varphi_{t}^{2} d x-k_{0} \int_{0}^{1}\left(w_{x}-l \varphi\right)^{2} d x+\rho_{1} \int_{0}^{1} w_{t}^{2} d x \\
& +k \int_{0}^{1}\left(\varphi_{x}+\psi+l w\right)^{2} d x+\frac{\rho_{1}}{2} \int_{0}^{1} \psi_{t}^{2} d x .
\end{aligned}
$$


Proof. A simple differentiation of $F_{6}$, using the first and third equations in (1), leads to

$$
\begin{aligned}
F_{6}^{\prime}(t)= & -\rho_{1} \int_{0}^{1} \varphi_{t}^{2} d x-k_{0} \int_{0}^{1}\left(w_{x}-l \varphi\right)^{2} d x+\rho_{1} \int_{0}^{1} w_{t}^{2} d x \\
& -\rho_{1} \int_{0}^{1} \varphi_{t} \int_{0}^{x} \psi_{t}(y) d y+k \int_{0}^{1}\left(\varphi_{x}+\psi+l w\right)^{2} d x \\
& +l\left(k-k_{0}\right) \int_{0}^{1}\left(w_{x}-l \varphi\right) \int_{0}^{x}\left(\varphi_{x}+\psi+l w\right) d y d x
\end{aligned}
$$

and using Young's and Cauchy-Schwarz inequalities, with the fact that $k=k_{0}$, gives (76).

Lemma 12. Let $\left(\varphi, \psi, w, \theta, q, z, \eta^{t}\right)$ be the solution of (19)-(21) and let (9) holds, and we have

$$
\begin{aligned}
F_{7}(t):= & \rho_{2} \int_{0}^{1} \psi_{t}\left(\varphi_{x}+\psi+l w\right) d x+\frac{b \rho_{1}}{k} \int_{0}^{1} \varphi_{t} \psi_{x} d x \\
& +\frac{b \rho_{3}}{\gamma}\left(\frac{\rho_{1}}{k}-\frac{\rho_{2}}{b}\right) \int_{0}^{1} \theta \varphi_{t} d x \\
& -\frac{b}{\gamma}\left(\frac{\rho_{1}}{k}-\frac{\rho_{2}}{b}\right) \int_{0}^{1} q\left(\varphi_{x}+\psi+l w\right) d x \\
& -\frac{b l^{2} \rho_{2}}{k_{0}} \int_{0}^{1} \psi \psi_{t} d x+\frac{b l \rho_{1}}{k_{0}} \int_{0}^{1} \psi w_{t} d x
\end{aligned}
$$

satisfies, for any $\varepsilon_{4}, \varepsilon_{5}, \delta_{3}>0$, the estimate

$$
\begin{aligned}
& F_{7}^{\prime}(t) \leq-\left(\frac{k}{2}-\frac{b \eta}{\gamma \alpha \varepsilon_{10}}+\frac{\gamma}{4 \varepsilon_{1}}+\frac{k b \rho_{3}}{\gamma 4 \varepsilon_{2} \rho_{1}}\left(\frac{\rho_{1}}{k}-\frac{\rho_{2}}{b}\right)\right. \\
& \left.+\frac{b}{4 \varepsilon_{3}}\right) \int_{0}^{1}\left(\varphi_{x}+\psi+l w\right)^{2} d x+\varepsilon_{8} \int_{0}^{1} w_{t}^{2} d x \\
& +\left(\frac{b^{2} l^{2}}{k}+\frac{b l^{2} \rho_{2} \delta_{3}}{k_{0}}+b \varepsilon_{3}+\frac{b l^{2}}{k_{0}} c_{1}\right. \\
& \left.+2\left(\frac{\varepsilon}{b^{2} \lambda_{1}}+\frac{b^{2}}{2 \varepsilon \lambda_{1}}\right)+c_{2}\right) \int_{0}^{1} \psi_{x}^{2} d x \\
& +\varepsilon_{9} \int_{0}^{1}\left(w_{x}-l \varphi\right)^{2} d x+c\left(1+\frac{1}{\varepsilon_{8}}+\frac{b \rho_{1} \varepsilon_{4}}{k}\right) \\
& \text { - } \int_{0}^{1} \psi_{t}^{2} d x+c\left(1+\frac{1}{\varepsilon_{8}}\right) \int_{0}^{1} q^{2} d x+c\left(1+\frac{1}{\varepsilon_{9}}\right. \\
& \left.+\frac{b \rho_{3} \mu_{1}}{\gamma \rho_{1}} \varepsilon_{5}\left(\frac{\rho_{1}}{k}-\frac{\rho_{2}}{b}\right)\right) \int_{0}^{1} \theta^{2} d x+\left(\frac{b \eta}{\gamma \alpha} \varepsilon_{10}\right. \\
& \left.+\gamma \varepsilon_{1}+\frac{k b \rho_{3}}{\gamma \rho_{1}} \varepsilon_{2}\left(\frac{\rho_{1}}{k}-\frac{\rho_{2}}{b}\right)\right) \int_{0}^{1} \theta_{x}^{2} d x \\
& +\left(\frac{b \rho_{1}}{k 4 \varepsilon_{4}}+\frac{b \rho_{3} \mu_{1}}{4 \varepsilon_{5} \gamma \rho_{1}}\left(\frac{\rho_{1}}{k}-\frac{\rho_{2}}{b}\right)\right) \int_{0}^{1} \varphi_{t}^{2} d x \\
& +\frac{l \varepsilon_{1}}{b^{2}} \int_{0}^{1}(w+\psi)^{2} d x+\frac{\varepsilon}{2} \int_{0}^{1}\left(\varphi_{x}+\psi\right)^{2} \\
& +\frac{g_{0} b l^{2} \rho_{2}}{k_{0} 4 \delta_{3}} \int_{0}^{1} \int_{0}^{\infty} g(s)\left|\eta_{x}^{t}(x, s)\right|^{2} d s d x .
\end{aligned}
$$

Proof. Taking the deviate of $F_{7}$, we obtain

$$
\begin{aligned}
F_{7}^{\prime}(t)= & \rho_{2} \int_{0}^{1} \psi_{t t}\left(\varphi_{x}+\psi+l w\right) d x+\rho_{2} \int_{0}^{1} \psi_{t}\left(\varphi_{x}+\psi+l w\right)_{t} d x \\
& +\frac{b \rho_{1}}{k} \int_{0}^{1} \varphi_{t t} \psi_{x} d \mathrm{x}-\frac{b \rho_{1}}{k} \int_{0}^{1} \varphi_{t} \psi_{x t} d x \\
& +\frac{b \rho_{3}}{\gamma}\left(\frac{\rho_{1}}{k}-\frac{\rho_{2}}{b}\right) \int_{0}^{1} \theta_{t} \varphi_{t} d x \\
& +\frac{b \rho_{3}}{\gamma}\left(\frac{\rho_{1}}{k}-\frac{\rho_{2}}{b}\right) \int_{0}^{1} \theta \varphi_{t t} d x \\
& -\frac{b}{\gamma}\left(\frac{\rho_{1}}{k}-\frac{\rho_{2}}{b}\right) \int_{0}^{1} q_{t}\left(\varphi_{x}+\psi+l w\right) d x \\
& -\frac{b}{\gamma}\left(\frac{\rho_{1}}{k}-\frac{\rho_{2}}{b}\right) \int_{0}^{1} q\left(\varphi_{x}+\psi+l w\right)_{t} d x \\
& -\frac{b l^{2} \rho_{2}}{k_{0}} \int_{0}^{1} \psi_{t}^{2} d x-\frac{b l^{2} \rho_{2}}{k_{0}} \int_{0}^{1} \psi_{t t} \psi d x \\
& +\frac{b l \rho_{1}}{k_{0}} \int_{0}^{1} w_{t t} \psi d x+\frac{b l \rho_{1}}{k_{0}} \int_{0}^{1} w_{t} \psi t d x
\end{aligned}
$$

From the RHS of (80) and the relations in (1)-(3), we arrive at

$$
\begin{array}{r}
\rho_{2} \int_{0}^{1} \psi_{t t}\left(\varphi_{x}+\psi+l w\right) d x \\
=-k \int_{0}^{1}\left(\varphi_{x}+\psi+l w\right)^{2} d x-\gamma \int_{0}^{1} \theta_{x}\left(\varphi_{x}+\psi+l w\right) d x \\
\quad-b \int_{0}^{1} \psi_{x}\left(\varphi_{x}+\psi+l w\right)_{x} d x \\
\quad-\int_{0}^{1} \int_{0}^{\infty} g(s) \psi_{x x}(x, t-s) d s\left(\varphi_{x}+\psi+l w\right) d x \\
\quad-\int_{0}^{1} f(\psi)\left(\varphi_{x}+\psi+l w\right) d x \\
\rho_{1} \int_{0}^{1} \varphi_{t t} \psi_{x} d x=k \int_{0}^{1} \psi_{x}\left(\varphi_{x}+\psi+l w\right)_{x} d x \\
+\int_{0}^{1} q_{t}\left(\varphi_{x}+\psi+l w\right) d x=\frac{\beta}{\alpha} \int_{0}^{1} q\left(\varphi_{x}+\psi+l w\right) d x \\
\quad+\frac{k k_{0}}{\rho_{1}} \int_{0}^{1} \theta\left(w_{x}-l \varphi\right) d x-\frac{\mu_{1}}{\rho_{1}} \int_{0}^{1} \theta \varphi_{t} d x \\
\rho_{3} \int_{0}^{1} \theta_{t} \varphi_{t} d x=k \int_{0}^{1} q \varphi_{x t} d x+\gamma \int_{0}^{1} \varphi_{t} \varphi_{x t} d x \\
\int_{0}^{1} \varphi_{t} d x=-\frac{k}{\rho_{1}} \int_{0}^{1} \theta_{x}\left(\varphi_{x}+\psi+l w\right) d x
\end{array}
$$




$$
\begin{array}{r}
-\rho_{2} \int_{0}^{1} \psi_{t t} \psi d x=b \int_{0}^{1} \psi_{x}^{2} d x+k \int_{0}^{1} \psi\left(\varphi_{x}+\psi+l w\right) d x \\
-\gamma \int_{0}^{1} \theta \psi_{x} d x+\int_{0}^{1} \int_{0}^{\infty} g(s) \psi_{x x} d s \psi d x \\
+\int_{0}^{1} f(\psi) \psi d x \\
\rho_{1} \int_{0}^{1} w_{t t} \psi d x=-k_{0} \int_{0}^{1} \psi_{x}\left(w_{x}-l \varphi\right) d x \\
-k l \int_{0}^{1} \psi\left(\varphi_{x}+\psi+l w\right) d x .
\end{array}
$$

Invoking to (81)-(87) into (80), we arrive at

$$
\begin{aligned}
F_{7}^{\prime}(t)= & -k \int_{0}^{1}\left(\varphi_{x}+\psi+l w\right)^{2} d x+\left(\rho_{2}-\frac{b l^{2} \rho_{2}}{k_{0}}\right) \int_{0}^{1} \psi_{t}^{2} d x \\
& +\left(l \rho_{2}+\frac{b l \rho_{1}}{k_{0}}\right) \int_{0}^{1} \psi_{t} w_{t} d x+\frac{b \eta}{\alpha \gamma} \int_{0}^{1} \theta_{x}\left(\varphi_{x}+\psi+l w\right) d x \\
& -\frac{b}{\gamma}\left(\frac{\rho_{1}}{k}-\frac{\rho_{2}}{b}\right) \int_{0}^{1} q \psi_{t} d x-\frac{b l}{\gamma}\left(\frac{\rho_{1}}{k}-\frac{\rho_{2}}{b}\right) \int_{0}^{1} q w_{t} d x \\
& +\frac{b l k_{0} \rho_{3}}{\gamma \rho_{1}}\left(\frac{\rho_{1}}{k}-\frac{\rho_{2}}{b}\right) \int_{0}^{1} \theta\left(w_{x}-l \varphi\right) d x-\frac{\gamma b l^{2}}{k_{0}} \int_{0}^{1} \theta \psi_{x} d x \\
& +\frac{b \beta}{\alpha \gamma}\left(\frac{\rho_{1}}{k}-\frac{\rho_{2}}{b}\right) \int_{0}^{1} q\left(\varphi_{x}+\psi+l w\right) d x+\frac{b^{2} l^{2}}{k_{0}} \int_{0}^{1} \psi_{x}^{2} d x \\
& -b l \int_{0}^{1} \psi_{x}\left(w_{x}-l \varphi\right) d x-\gamma \int_{0}^{1} \theta_{x}\left(\varphi_{x}+\psi+l w\right) d x \\
& -\frac{k b \rho_{3}}{\gamma \rho_{1}}\left(\frac{\rho_{1}}{k}-\frac{\rho_{2}}{b}\right) \int_{0}^{1} \theta_{x}\left(\varphi_{x}+\psi+l w\right) d x \\
& -b \int_{0}^{1} \psi_{x}\left(\varphi_{x}+\psi+l w\right)_{x} d x+\frac{b \rho_{1}}{k} \int_{0}^{1} \psi \varphi_{x t} d x \\
& -\frac{b \rho_{3} \mu_{1}}{\gamma \rho_{1}}\left(\frac{\rho_{1}}{k}-\frac{\rho_{2}}{b}\right) \int_{0}^{1} \theta \varphi_{t} d x-\int_{0}^{1} f(\psi)\left(\varphi_{x}+\psi+l w\right) d x \\
& +\frac{b l^{2}}{k_{0}} \int_{0}^{1} f(\psi) \psi d x+\frac{b l^{2} \rho_{2}}{k_{0}} \int_{0}^{1} \psi_{x}(x) \int_{0}^{\infty} g(s) \eta_{x}^{t}(x, s) \cdot d s d x .
\end{aligned}
$$

We thus have

$$
\begin{aligned}
\int_{0}^{1}\left|\varphi_{x} f(\psi)\right| d x & \leq\left\|\varphi_{x}\right\|\|\psi\|_{2(\theta+1)}^{\theta}\|\psi\|_{2(\theta+1)} \\
& \leq \frac{\varepsilon}{2 b^{2}} \int_{0}^{1} \varphi_{x}^{2} d x+\frac{b^{2}}{2 \varepsilon \lambda_{1}} \int_{0}^{1} \psi_{x}^{2} d x \\
& \leq \frac{\varepsilon}{b^{2}} \int_{0}^{1}\left(\varphi_{x}+\psi\right)^{2} d x+\frac{\varepsilon}{b^{2}} \int_{0}^{1} \psi^{2} d x+\frac{b^{2}}{2 \varepsilon \lambda_{1}} \int_{0}^{1} \psi_{x}^{2} d x \\
& \leq \frac{\varepsilon}{b^{2}} \int_{0}^{1}\left(\varphi_{x}+\psi\right)^{2} d x+\left(\frac{\varepsilon}{b^{2} \lambda_{1}}+\frac{b^{2}}{2 \varepsilon \lambda_{1}}\right) \int_{0}^{1} \psi_{x}^{2} d x
\end{aligned}
$$

Estimate (79) follows thanks to Young's inequality and the fact that $k=k_{0}$.
Lemma 13. Let $\left(\varphi, \psi, w, \theta, q, z, \eta^{t}\right)$ be the solution of (19)-(21). Then, the energy functional

$$
F_{8}(t):=\int_{0}^{1} \rho_{1} \varphi_{t} \varphi d x+\frac{\mu_{1}}{2} \int_{0}^{1} \varphi^{2} d x
$$

Then, we have the following estimate, for any $\varepsilon_{11}>0$,

$$
\begin{aligned}
F_{8}^{\prime}(t) \leq & \left(-K+\varepsilon_{11}\left(\frac{K}{2}+\frac{\mu_{2} c}{2}\right)\right) \int_{0}^{1} \varphi_{x}^{2} d x+\frac{K}{2 \varepsilon_{11}} \int_{0}^{1} \psi_{x}^{2} d x \\
& +\frac{\mu_{2}}{2 \varepsilon_{11}} \int_{0}^{1} z^{2}(x, 1, t) d x+\rho_{1} \int_{0}^{1} \varphi_{t}^{2} d x,
\end{aligned}
$$

where $c=1 / \pi^{2}$ is the Poincaré constant.

Proof. Taking the derivative of (90) with respect to $t$, we have

$$
F_{8}^{\prime}(t)=\rho_{1} \int_{0}^{1} \varphi_{t t} \varphi d x+\rho_{1} \int_{0}^{1} \varphi_{t}^{2} d x+\mu_{1} \int_{0}^{1} \varphi_{t} \varphi d x
$$

Then, by using the first equation in (1), we find

$F_{8}^{\prime}(\mathrm{t})=k \int_{0}^{1}\left(\varphi_{x}+\psi+l w\right)_{x} \varphi d x-\mu_{2} \int_{0}^{1} \varphi z(x, 1, t) d x+\rho_{1} \int_{0}^{1} \varphi_{t}^{2} d x$.

Consequently, we arrive at

$$
F_{8}^{\prime}(t)=-k \int_{0}^{1}\left(\varphi_{x}+\psi+l w\right) \varphi_{x} d x-\mu_{2} \int_{0}^{1} \varphi z(x, 1, t) d x+\rho_{1} \int_{0}^{1} \varphi_{t}^{2} d x
$$

Applying Young's inequality and Poincaré's inequality, we find (90).

Lemma 14. Let $\left(\varphi, \psi, w, \theta, q, z, \eta^{t}\right)$ be the solution of (19)-(21). Then, we define the functional

$$
F_{9}(t):=\int_{0}^{1} \int_{0}^{1} e^{-2 \tau \rho} z^{2}(x, \rho, t) d \rho d x
$$

Then, the following result holds.

$$
F_{9}^{\prime}(t) \leq-F_{9}(t)-\frac{c_{1}}{2 \tau} \int_{0}^{1} z^{2}(x, 1, t) d x+\frac{1}{2 \tau} \int_{0}^{1} \psi_{t}^{2}(x, t) d x,
$$

where $c$ is a positive constant. 
Proof. Taking the deviate of (95) with respect to $t$ and using the equation (16), we get

$$
\begin{aligned}
& \frac{d}{d t}\left(\int_{0}^{1} \int_{0}^{1} e^{-2 \tau \rho} z^{2}(x, \rho, t) d \rho d x\right) \\
& =-\frac{1}{\tau} \int_{0}^{1} \int_{0}^{1} e^{-2 \tau \rho} z z_{\rho}(x, \rho, t) d \rho d x \\
& =-\int_{0}^{1} \int_{0}^{1} e^{-2 \tau \rho} z^{2}(x, \rho, t) d \rho d x \\
& \quad-\frac{1}{2 \tau} \int_{0}^{1} \int_{0}^{1} \frac{\partial}{\partial \rho}\left(e^{-2 \tau \rho} z^{2}(x, \rho, t)\right) d \rho d x .
\end{aligned}
$$

Making use of the estimate above, implies that there exists a positive constant $c_{1}$ such that (96) holds.

Theorem 15. Assume that $\eta=0$ and $k=k_{0}$. Then, $(\varphi, \psi, w$, $\left.\theta, q, z, \eta^{t}\right)$ the solution of (19)-(21) satisfies

$$
E(t) \leq c_{0} e^{-c_{1} t}, t \geq 0,
$$

where the positive constant $c_{0}$ is directly depending on initial data and the uniform constant $c_{1}$ is depending only on the coefficients of the system. For $N, N_{i}>0$,

$$
\mathscr{L}(t):=N E(t)+\sum_{i=1}^{i=9} N_{i} F_{i}(t)
$$

Then, from (56), (59), (62), (66), (69), (73), (76), (79), (91), and (96), we have

$$
\begin{aligned}
\mathscr{L}^{\prime}(t) \leq & \left.-\beta N+c_{1}\left(1+\frac{1}{\varepsilon_{1}}\right)+c N_{2}+c\left(1+\frac{1}{\varepsilon_{8}}\right) N_{7}\right] \int_{0}^{1} q^{2} d x \\
& -N\left[\mu_{1}-\frac{\xi}{2}-\frac{\left|\mu_{2}\right|}{2}\right]\left\|\varphi_{t}\right\|_{2}^{2}-N\left[\frac{\xi}{2}-\frac{\left|\mu_{2}\right|}{2}\right]\|z(x, 1, t)\|_{2}^{2} \\
& -\left[\frac{N_{1} \rho_{3}}{2}-N_{2}\left(C_{1}+1+\frac{1}{\varepsilon_{2}}+\frac{1}{\varepsilon_{3}}\right)-c N_{4}\right. \\
& \left.-c\left(1+\frac{1}{\varepsilon_{9}}+\frac{b \rho_{3} \mu_{1}}{\gamma \rho_{1} c} \varepsilon_{5}\left(\frac{\rho_{1}}{k}-\frac{\rho_{2}}{b}\right)\right) N_{7}\right] \int_{0}^{1} \theta^{2} d x \\
& +\left[\varepsilon_{1} N_{1}-C N-N_{2} \frac{\rho_{2}}{\gamma}+\rho_{2} N_{4}+c N_{5}+\frac{\rho_{1}}{2} N_{6}\right. \\
& \left.+c\left(1+\frac{1}{\varepsilon_{8}}+\frac{b \rho_{1} \varepsilon_{4}}{k}\right) N_{7}+\rho_{1} N_{8}+\frac{1}{2 \tau} N_{9}\right] \int_{0}^{1} \psi_{t}^{2} d x \\
& +\left[\varepsilon_{2} N_{2}+c N_{3}+\frac{k^{2}}{b} N_{4}+l k N_{5}+k N_{6}\right. \\
& -\left(\frac{k}{2}-\frac{b \tilde{\eta}}{\alpha \gamma \varepsilon_{10}}+\frac{\gamma}{4 \varepsilon_{1}}+\frac{k b \rho_{3}}{\gamma 4 \varepsilon_{2} \rho_{1}}\left(\frac{\rho_{1}}{k}-\frac{\rho_{2}}{b}\right)\right. \\
& \left.\left.+\frac{b}{4 \varepsilon_{3}}\right) N_{7}\right] \int_{0}^{1}\left(\varphi_{x}+l w+\psi\right)^{2} d x
\end{aligned}
$$

$$
\begin{aligned}
& +\left[\left(\varepsilon_{3}+\frac{\rho_{3}}{\gamma}\left(\frac{\varepsilon_{2}}{b^{2} \lambda_{2}}+\frac{b^{2}}{2 \varepsilon_{2} \lambda_{2}}\right)\right) N_{2}+c N_{3}\right. \\
& +\left(\delta_{2}+\frac{b}{2}+C_{2}\right) N_{4}+\left(\frac{b l^{2} \rho_{2} \delta_{3}}{k_{0}}+\frac{b^{2} l^{2}}{k}+b \varepsilon_{3}\right. \\
& \left.+\frac{b l^{2}}{k_{0}} c_{1}+2\left(\frac{\varepsilon}{b^{2} \lambda_{1}}+\frac{b^{2}}{2 \varepsilon \lambda_{1}}\right)+c_{2}\right) N_{7} \\
& \left.+\left(\frac{k}{2 \varepsilon_{11}}-k+\varepsilon_{11} \frac{k}{2}+\varepsilon_{11} \frac{\mu_{2} c}{2}\right) N_{8}\right] \int_{0}^{1} \psi_{x}^{2} d x \\
& +\left[-\rho_{1} N_{3}-\frac{l \rho_{1}}{2} N_{5}+\rho_{1} N_{6}+\varepsilon_{8} N_{7}\right] \int_{0}^{1} w_{t}^{2} d x \\
& +\left[k_{0} N_{3}-\left(l k_{0}-\frac{\mu_{1}}{4 \varepsilon_{6}}-\frac{\mu_{2}}{4 \varepsilon_{7}}\right) N_{5}-k_{0} N_{6}+\varepsilon_{9} N_{7}\right] \\
& \cdot \int_{0}^{1}\left(w_{x}-l \varphi\right)^{2} d x+\left[\frac{\mu_{2}}{4 \varepsilon_{5}} N_{3}+\varepsilon_{7} \mu_{2} N_{5}+\frac{\mu_{2}}{2 \varepsilon_{11}} N_{8}-\frac{c_{1}}{2 \tau} N_{9}\right] \\
& \text {. } \int_{0}^{1} z^{2}(x, 1, t) d x+\left[\varepsilon_{5} \mu_{2}+\varepsilon_{4} \mu_{1}\right] N_{3} \int_{0}^{1} \varphi^{2} d x \\
& +\left[-\left(1-\frac{1}{4 \varepsilon_{4}}\right) N_{3}+\left(l \rho_{1}+\varepsilon_{6} \mu_{1}\right) N_{5}-\frac{\rho_{1}}{2} N_{6}\right. \\
& \left.+\rho_{1} N_{8}+\left(\frac{b \rho_{1}}{k 4 \varepsilon_{4}}+\frac{b \rho_{3} \mu_{1}}{4 \varepsilon_{5} \gamma \rho_{1}}\left(\frac{\rho_{1}}{k}-\frac{\rho_{2}}{b}\right)\right) N_{7}\right] \int_{0}^{1} \varphi_{t}^{2} d x \\
& +\left[\left(-k+\varepsilon_{11}\left(\frac{k}{2}+\frac{\mu_{2} c}{2}\right)\right) N_{8}\right] \int_{0}^{1} \varphi_{x}^{2} d x \\
& +\left[\left(\frac{b \tilde{\eta} \varepsilon_{10}}{\alpha \gamma}+\gamma \varepsilon_{1}+\frac{k b \rho_{3}}{\gamma \rho_{1}} \varepsilon_{2}\left(\frac{\rho_{1}}{k}-\frac{\rho_{2}}{b}\right)\right) N_{7}\right. \\
& \left.+N_{2} \delta_{1}\right] \int_{0}^{1} \theta_{x}^{2} d x-N_{9} F_{9}(t)+\left(\frac{N_{2} g_{0}}{4 \delta_{1}}+\frac{N_{4} g_{0}}{4 \delta_{2}}\right. \\
& \left.+\frac{N_{7} g_{0} b l^{2} \rho_{2}}{k_{0} 4 \delta_{3}}-N \frac{\zeta}{2}\right) \int_{0}^{1} \int_{0}^{\infty} g(s)\left|\eta_{x}^{t}(x, s)\right|^{2} d s d x
\end{aligned}
$$

At this point, we have to choose our constants very carefully. First, choosing $\varepsilon_{i} i=1, \ldots 10$ small enough such that

$$
\varepsilon_{1} \leq \frac{N_{2}\left(\rho_{2} / \gamma\right)+\rho_{2} N_{4}+c N_{5}+\left(\rho_{1} / 2\right) N_{6}}{N_{1}} .
$$

Moreover, we pick $N_{9}$ large enough so that

$$
\begin{gathered}
\frac{\mu_{2}}{4 \varepsilon_{5}} N_{3}+\varepsilon_{7} \mu_{2} N_{5}+\frac{\mu_{2}}{2 \varepsilon_{11}} N_{8}-\frac{c_{1}}{2 \tau} N_{9} \leq 0, \\
N_{9} \geq \frac{\left(\mu_{2} / 4 \varepsilon_{5}\right) N_{3}+\varepsilon_{7} \mu_{2} N_{5}+\left(\mu_{2} / 2 \varepsilon_{11}\right) N_{8}}{c_{1} / 2 \tau},
\end{gathered}
$$

and we take $\varepsilon_{11}$ small enough such that

$$
\varepsilon_{11} \leq \frac{k}{\left(k / 2+\mu_{2} c / 2\right) N_{8}}
$$


Next, choosing $N_{5}$ large enough such that

$$
\frac{N_{5} \rho_{3} \kappa}{4} \geq N_{4}\left(\gamma \rho_{3}+\frac{\rho_{3}}{2 \varepsilon_{4}}(b+2 \kappa)\right) .
$$

After that, we can choose $N$ large enough such that

$$
\begin{gathered}
N \geq \frac{c_{1}\left(1+1 / \varepsilon_{1}\right)+c N_{2}+c\left(1+1 / \varepsilon_{8}\right) N_{7}}{\beta}, \\
\frac{N_{2} g_{0}}{4 \delta_{1}}+\frac{N_{4} g_{0}}{4 \delta_{2}}+\frac{g_{0} b l^{2} \rho_{2}}{k_{0} 4 \delta_{3}}-N \frac{\zeta}{2} \leq 0 .
\end{gathered}
$$

Thus, the relation (100) becomes

$$
\begin{aligned}
\frac{d}{d t} \mathscr{L}(t) \leq & -\eta_{1} \int_{0}^{1}\left(\psi_{t}^{2}+\psi_{x}^{2}+\varphi_{t}^{2}+\left(\varphi_{x}+l w+\psi\right)^{2}\right. \\
& \left.+\theta^{2}+q^{2}\right) d x-\eta_{1} \int_{0}^{1} \int_{0}^{1} z^{2}(x, \rho, t) d \rho d x,
\end{aligned}
$$

which leads by (55) that there exists also $\eta_{2}$, such that

$$
\frac{d}{d t} \mathscr{L}(t) \leq-\eta_{2} E(t), \forall t \geq 0
$$

Lemma 16. For $N$ large enough, there exist two positive constants $\beta_{1}$ and $\beta_{2}$ depending on $N_{i}, i=1, \cdots, 9$ and $\varepsilon_{i}, i=1, \cdots$ , 11 such that

$$
\beta_{1} E(t) \leq \mathscr{L}(t) \leq \beta_{2} E(t), \forall t \geq 0 .
$$

Proof. We consider the functional

$$
H(t)=\sum_{i=1}^{i=9} N_{i} F_{i}(t)
$$

and show that

$$
|H(t)| \leq C E(t), C>0 .
$$

From (58), (61), (65), (68), (72), (75), (78), (90), and (95), we obtain

$$
\begin{aligned}
|H(t)| \leq & N_{1}\left|\alpha \rho_{3} \int_{0}^{1} \theta \int_{0}^{x} q(y) d y d x\right| \\
& +N_{2}\left|-\frac{\rho_{2} \rho_{3}}{\gamma} \int_{0}^{1} \theta d x \int_{0}^{x} \psi_{t}(y) d y d x\right| \\
& +N_{3}\left|\rho_{1} \int_{0}^{1}\left(\varphi \varphi_{t}+w w_{t}\right) d x\right|
\end{aligned}
$$

$$
\begin{aligned}
& +N_{4}\left|\rho_{2} \int_{0}^{1} \int_{0}^{x} \psi \psi_{t}(t, x) d x\right| \\
& +N_{5}\left|-\rho_{1} \int_{0}^{1} \varphi_{t}\left(w_{x}-l \varphi\right) d x-\rho_{1} \int_{0}^{1} w_{t}\left(\varphi_{x}+\psi+l w\right) d x\right| \\
& +N_{6} \mid-\rho_{1} \int_{0}^{1}\left(w_{x}-l \varphi\right) \int_{0}^{x} w_{t}(y) d y d x \\
& -\rho_{1} \int_{0}^{1} \varphi_{t} \int_{0}^{x}\left(\varphi_{x}+\psi+l w\right) d y d x \mid \\
& +N_{7}\left|\rho_{2} \int_{0}^{1}\left(\varphi_{x}+\psi+l w\right) d x+\frac{b \rho_{1}}{k} \int_{0}^{1} \varphi_{t} \psi_{x} d x\right| \\
& +N_{8}\left|\int_{0}^{1} \rho_{1} \varphi \varphi_{t} d x+\frac{\mu_{1}}{2} \int_{0}^{1} \varphi^{2} d x\right| \\
& +N_{9} \int_{0}^{1} \int_{0}^{1} e^{-2 \tau \rho} z^{2}(x, \rho, t) d \rho d x .
\end{aligned}
$$

By using, the trivial relation

$$
\int_{0}^{1}(\varphi+l w)^{2} d x \leq 2 c \int_{0}^{1}\left(\varphi_{x}+l w+\psi\right)^{2} d x+2 c \int_{0}^{1} \psi_{x}^{2} d x
$$

Young's and Poincaré's inequalities, we get

$$
\begin{aligned}
|H(t)| \leq & \alpha_{1} \int_{0}^{1} \phi_{t}^{2} d x+\alpha_{2} \int_{0}^{1} \psi_{t}^{2} d x+\alpha_{3} \int_{0}^{1} w_{t}^{2} \\
& +\alpha_{4} \int_{0}^{1} \psi_{x}^{2}+\alpha_{5} \int_{0}^{1} \theta^{2} d x+\alpha_{6} \int_{0}^{1} q^{2} d x \\
& +\alpha_{7} \int_{0}^{1}\left(\left(\varphi_{x}+l w+\psi\right)^{2}+\left(w_{x}-l \varphi\right)^{2}\right) d x \\
& +\int_{0}^{1} \int_{0}^{1} z^{2}(x, \rho, t) d \rho d x
\end{aligned}
$$

where $\alpha_{1}, \cdots, \alpha_{6}$ are the positive constants as follows:

$$
\left\{\begin{array}{l}
\alpha_{1}:=\frac{1}{2}\left(N_{3} \rho_{1}+N_{8} \rho_{1}\right), \\
\alpha_{2}:=\frac{1}{2}\left(N_{4} \rho_{2}+N_{2} \frac{\rho_{2} \rho_{3}}{\gamma}\right), \\
\alpha_{3}=\frac{1}{2}\left(N_{3} \rho_{1}+N_{6} \rho_{1}\right), \\
\alpha_{4}:=\frac{b \rho_{1}}{2 k}, \\
\alpha_{5}:=\frac{1}{2}\left(N_{1} \rho_{3}+\frac{\rho_{2} \rho_{3}}{\gamma}\right), \\
\alpha_{6}:=\frac{1}{2}\left(N_{1} \rho_{3}+N_{5} \tau_{0} \rho_{3}\right), \\
\alpha_{7}:=\frac{1}{2}\left(N_{7} \rho_{2}+3 \rho_{1}\right) .
\end{array}\right.
$$


From (113), we have

$$
|H(t)| \leq \widehat{C} E(t)
$$

for

$$
\widehat{C}=\frac{\max \left\{\alpha_{1}, \alpha_{2}, \alpha_{3}, \alpha_{4}, \alpha_{5}, \alpha_{6}\right\}}{\min \left\{\rho_{1}, \rho_{2}, \rho_{3}, k, b, \kappa, \gamma, \delta, \tau_{0}\right\}} .
$$

Therefore, we get

$$
|\mathscr{L}(t)-N E(t)| \leq \widehat{C} E(t) .
$$

Then, we can choose $N$ large enough so that $\beta_{1}=N-\widehat{C}$ $>0$. Then, (108) holds true for $\beta_{2}=N+\widehat{C}>0$, and this concludes the proof of the Lemma.

Combining now (107) and (108), we conclude that there exists some $\Lambda>0$ such that

$$
\frac{\mathrm{d}}{d t} \mathscr{L}(t) \leq-\Lambda \mathscr{L}(t), \forall t \geq 0
$$

Integration of (118) yields

$$
\mathscr{L}(t) \leq \mathscr{L}(0) e^{-\Lambda t}, \forall t \geq 0 .
$$

Finally, using (108) and (119), so (98) is satisfied, we thus immediately reach to Theorem 15 .

\section{Conclusion and Perspective}

In this current study, a one-dimensional linear thermoelastic Bresse system with delay term, forcing, and infinity history acting on the shear angle displacement is considered. According to an appropriate assumption between the weight of the delay and the weight of the damping, the wellposedness of the problem using the semigroup method is proved, where an asymptotic stability result of global solution is obtained. In next article, we will generalize this result to convex bounded domain with a holomorphic map, and let $x$ and $y$ be two distinct fixed points for our problem. We will suppose there is at least one complex geodesics passing through two distinct variables. We will see that this method of proof cannot be generalized to the case of a bounded domain of a complex Banach space. Also, in the last part of the next article, we will study the fixed points of the analytical automorphisms of the open unit-ball B of a complex Banach space. More precisely, we will assume that $B$ is homogeneous and we will show that, if the right hand side is an analytical automorphism of $\mathrm{B}$, there exists a complex geodesic which we will specify formed of fixed points of the right hand. We will see that the set of fixed points of the right hand can be much larger by using the studied algorithm in ([46-51]).

\section{Data Availability}

No data were used to support the study.

\section{Conflicts of Interest}

The authors declare that they have no competing interests.

\section{Acknowledgments}

The fourth-named author extends their appreciation to the Deanship of Scientific Research at King Khalid University for funding work through research group program under grant R.G.P.2/1/42.

\section{References}

[1] A. Choucha, S. Boulaaras, D. Ouchenane, B. Cherif, and M. Abdalla, "Exponential stability of swelling porous elastic with a viscoelastic damping and distributed delay term," Journal of Function Spaces, vol. 2021, Article ID 5581634, 8 pages, 2021.

[2] S. Boulaaras, A. Draifia, and K. Zennir, "General decay of nonlinear viscoelastic Kirchhoff equation with BalakrishnanTaylor damping and logarithmic nonlinearity," Mathematical Methods in the Applied Sciences, vol. 42, no. 14, pp. 47954814, 2019.

[3] S. Boulaaras, A. Choucha, B. Cherif, A. Alharbi, and M. Abdalla, "Blow up of solutions for a system of two singular nonlocal viscoelastic equations with damping, general source terms and a wide class of relaxation functions," AIMS Mathematics, vol. 6, no. 5, pp. 4664-4676, 2021.

[4] A. Choucha, D. Ouchenane, and S. Boulaaras, "Well posedness and stability result for a thermoelastic laminated Timoshenko beam with distributed delay term," Mathematical Methods in the Applied Sciences, vol. 43, no. 17, pp. 9983-10004, 2020.

[5] A. Choucha, S. M. Boulaaras, D. Ouchenane, S. Alkhalaf, I. Mekawy, and M. Abdalla, "On the system of Coupled nondegenerate Kirchhoff equations with distributed delay: global existence and exponential decay," Journal of Function Spaces, vol. 2021, Article ID 5577277, 13 pages, 2021.

[6] N. H. Sweilam, A. A. Elaziz el-Sayed, and S. Boulaaras, "Fractional-order advection-dispersion problem solution via the spectral collocation method and the non-standard finite difference technique," Chaos, Solitons Fractals, vol. 144, article 110736, 2021.

[7] A. Choucha, S. Boulaaras, and D. Ouchenane, "Exponential decay of solutions for a viscoelastic coupled lame system with logarithmic source and distributed delay terms," Mathematical Methods in the Applied Sciences, vol. 44, no. 6, pp. 4858-4880, 2021.

[8] N. Mezouar, S. M. Boulaaras, and A. Allahem, "Global existence of solutions for the viscoelastic Kirchhoff equation with logarithmic source terms," Complexity, vol. 2020, Article ID 7105387, 25 pages, 2020.

[9] A. Choucha, S. Boulaaras, D. Ouchenane, S. Alkhalf, and B. Cherif, "Stability result and well-posedness for Timoshenko's beam laminated with thermoelastic and past history," Fractals, vol. 29, article 2140025, 2021.

[10] N. Mezouar and S. Boulaaras, "Global existence and exponential decay of solutions for generalized coupled non-degenerate Kirchhoff system with a time varying delay term," Boundary Value Problems, vol. 2020, no. 1, 2020.

[11] A. A. Keddi, A. T. Apalara, and S. A. Messaoudi, "Exponential and polynomial decay in a Thermoelastic-Bresse system with 
second sound," Applied Mathematics and Optimization, vol. 77, no. 2, pp. 315-341, 2018.

[12] V. Komornik, Exact Controllability and Stabilization. The Multiplier Method, RAM: Research in Applied Mathematics, John Wiley \& Sons, Ltd., Chichester, Masson, Paris, 1994.

[13] A. J. Muñoz-Vázquez, J. D. Sánchez-Torres, M. Defoort, and S. Boulaaras, "Predefined-time convergence in fractionalorder systems," Chaos, Solitons Fractals, vol. 143, article 110571, 2021.

[14] A. Rahmoune, D. Ouchenane, S. Boulaaras, and P. Agarwal, "Growth of solutions for a coupled nonlinear Klein-Gordon system with strong damping, source, and distributed delay terms," Advances in Difference Equations, vol. 2020, no. 1, 2020.

[15] S. Boulaaras and N. Doudi, "Global existence and exponential stability of coupled Lamé system with distributed delay and source term without memory term," Boundary Value Problems, vol. 2020, no. 1, 2020.

[16] S. Boulaaras, "Solvability of the Moore-Gibson-Thompson equation with viscoelastic memory term and integral condition via Galerkin method," Fractals, vol. 29, no. 5, article 2140021, p. 18, 2021.

[17] A. S. Nicaise and C. Pignotti, "Stabilization of the wave equation with boundary or internal distributed delay," Differential and Integral Equations, vol. 21, no. 9-10, pp. 935-958, 2008.

[18] C. Q. Xu, S. P. Yung, and L. K. Li, "Stabilization of wave systems with input delay in the boundary control," ESAIM: Control, Optimisation and Calculus of Variations, vol. 12, no. 4, pp. 770-785, 2006.

[19] R. Guefaifia, S. M. Boulaaras, A. A. E. el-Sayed, M. Abdalla, and B. Cherif, "On existence of sequences of weak solutions of fractional systems with Lipschitz nonlinearity," Journal of Function Spaces, vol. 2021, Article ID 5510387, 12 pages, 2021.

[20] F. Kamache, S. M. Boulaaras, R. Guefaifia, N. T. Chung, B. Cherif, and M. Abdalla, "On existence of multiplicity of weak solutions for a new class of nonlinear fractional boundary value systems via variational approach," Advances in Mathematical Physics, vol. 2021, Article ID 5544740, 10 pages, 2021.

[21] N. Doudi, S. M. Boulaaras, A. M. Alghamdi, and B. Cherif, "Polynomial decay rate for a coupled Lamé system with viscoelastic damping and distributed delay terms," Journal of Function Spaces, vol. 2020, Article ID 8879366, 14 pages, 2020.

[22] S. Boulaaras and N. Mezaour, "Global existence and decay of solutions of a singular nonlocal viscoelastic system with a nonlinear source term, nonlocal boundary condition, and localized damping term," Mathematical Methods in the Applied Sciences, vol. 43, no. 10, pp. 6140-6164, 2020.

[23] J. A. C. Bresse, Cours de Méchanique Appliquée, Mallet Bachelier, Paris, 1859.

[24] S. Boulaaras, F. Kamache, Y. Bouizem, and R. Guefaifia, “General decay and blow-up of solutions for a nonlinear wave equation with memory and fractional boundary damping terms," Boundary Value Problems, vol. 2020, no. 1, 2020.

[25] J. U. Kim and Y. Renardy, "Boundary control of the Timoshenko beam," SIAM Journal on Control and Optimization, vol. 25, no. 6, pp. 1417-1429, 1987.

[26] N. Mezouar and S. Boulaaras, "Global existence and decay of solutions of a singular nonlocal viscoelastic system with damping terms," Topological Methods in Nonlinear Analysis, vol. 56, no. 1, pp. 1-312, 2020.
[27] N. Mezouar and S. Boulaaras, "Global existence and decay of solutions for a class of viscoelastic Kirchhoff equation," Bulletin of the Malaysian Mathematical Sciences Society, vol. 43, no. 1, pp. 725-755, 2020.

[28] J. E. Munoz Rivera and R. Racke, "Global stability for damped Timoshenko systems," Discrete \& Continuous Dynamical Systems - A, vol. 9, no. 6, pp. 1625-1639, 2003.

[29] N. Doudi and S. Boulaaras, "Global existence combined with general decay of solutions for coupled Kirchhoff system with a distributed delay term," RACSAM, vol. 114 , no. 4 , p. 204, 2020.

[30] D. Ouchenane, A. Choucha, M. Abdalla, S. M. Boulaaras, and B. Cherif, "On the porous-elastic system with thermoelasticity of type III and distributed delay: well-posedness and stability," Journal of Function Spaces, vol. 2021, Article ID 9948143, 12 pages, 2021.

[31] D. Ouchnene, "A stability result of a Timoshenko system in thermoelasticity of second sound with a delay term in the internal feedback," Georgian Mathematical Journal, vol. 21, no. 4, pp. 475-489, 2014.

[32] J. H. Park and J. R. Kang, "Energy decay of solutions for Timoshenko beam with a weak non-linear dissipation," IMA Journal of Applied Mathematics, vol. 76, no. 2, pp. 340-350, 2011.

[33] C. A. Raposo, J. Ferreira, M. L. Santos, and N. N. O. Castro, "Exponential stability for the Timoshenko system with two weak dampings," Applied Mathematics Letters, vol. 18, no. 5, pp. 535-541, 2005.

[34] S. P. Timoshenko, "LXVI.On the correction for shear of the differential equation for transverse vibrations of prismatic bars," Philosophical Magazine, vol. 41, no. 245, pp. 744-746, 1921.

[35] F. Alabau Boussouira, J. E. Munôz Rivera, and D. S. Almeida Junior, "Stability to weak dissipative Bresse system," Journal of Mathematical Analysis and Applications, vol. 374, no. 2, pp. 481-498, 2011.

[36] A. Choucha, D. Ouchenane, S. Boulaaras, B. Cherif, and M. Abdalla, "Well-posedness and stability result of the nonlinear thermodiffusion full von Kármán beam with thermal effect and time-varying delay," Journal of Function Spaces, vol. 2021, Article ID 9974034, 16 pages, 2021.

[37] Z. Liu and B. Rao, "Energy decay rate of the thermoelastic Bresse system," Zeitschrift für Angewandte Mathematik und Physik, vol. 60, pp. 54-69, 2009.

[38] D. Ouchenane, S. Boulaaras, and F. Mesloub, "General decay for a viscoelastic problem with not necessarily decreasing kernel," Applicable Analysis, vol. 98, no. 9, pp. 1677-1693, 2018.

[39] M. L. Santos, A. Soufyane, and D. S. Almeida Junior, "Asymptotic behavior to Bresse system with past history," Quarterly of Applied Mathematics, vol. 73, no. 1, pp. 23-54, 2015.

[40] S. Toualbia, A. Zaraï, and S. Boulaaras, "Decay estimate and non-extinction of solutions of p-Laplacian nonlocal heat equations," AIMS Mathematics, vol. 5, no. 3, pp. 1663-1679, 2020.

[41] M. O. Alves, L. H. Fatori, J. Silva, and R. N. Monteiro, "Stability and optimality of decay rate for a weakly dissipative Bresse system," Mathematical Methods in the Applied Sciences, vol. 38, no. 5, pp. 898-908, 2015.

[42] L. H. Fatori and J. E. Munoz Rivera, "Rates of decay to weak thermoelastic Bresse system," IMA Journal of Applied Mathematics, vol. 75, no. 6, pp. 881-904, 2010.

[43] J. A. Soriano, J. E. Muñoz Rivera, and L. H. Fatori, "Bresse system with indefinite damping," Journal of Mathematical Analysis and Applications, vol. 387, no. 1, pp. 284-290, 2012. 
[44] A. Wehbe and W. Youssef, "Exponential and polynomial stability of an elastic Bresse system with two locally distributed feedbacks," Journal of Mathematical Physics, vol. 51, no. 10, article 103523, 2010.

[45] A. Pazy, Semigroups of Linear Operators and Applications to Partial Differential Equations, Vol 44 of Applied Math. Sciences, Springer-Verlag, New York, NY, USA, 1983.

[46] S. Boulaaras and M. Haiour, "The finite element approximation of evolutionary Hamilton-Jacobi-Bellman equations with nonlinear source terms," Indagationes Mathematicae, vol. 24, no. 1, pp. 161-173, 2013.

[47] S. Boulaaras, L. M. A. Bencheikh, and M. Haiour, "A new error estimate on uniform norm of a parabolic variational inequality with nonlinear source terms via the subsolution concepts," Journal of Inequalities and Applications, vol. 2020, no. 1, 18 pages, 2020.

[48] S. Boulaaras, M. S. Touati Brahim, S. Bouzenada, and A. Zarai, "An asymptotic behavior and a posteriori error estimates for the generalized Schwartz method of advection-diffusion equation," Acta Mathematica Scientia, vol. 38, no. 4, pp. 12271244, 2018.

[49] S. Boulaaras and M. Haiour, " $L^{\infty}$-asymptotic behavior for a finite element approximation in parabolic quasi-variational inequalities related to impulse control problem," Applied Mathematics and Computation, vol. 217, no. 14, pp. 64436450, 2011.

[50] S. Boulaaras, "Some new properties of asynchronous algorithms of theta scheme combined with finite elements methods for an evolutionary implicit 2-sided obstacle problem," Mathematical Methods in the Applied Sciences, vol. 40, no. 18 , pp. 7231-7239, 2017.

[51] S. Boulaaras and M. Haiour, "A new proof for the existence and uniqueness of the discrete evolutionary HJB equations," Applied Mathematics and Computation, vol. 262, pp. 42-55, 2015. 